\title{
Vegetation variation trend and its influencing factors in Urad Grassland over remote sensing
}

\author{
Yueying Zhang, Tiantian Liu, Yuxi Wang, Ming Zhang and Yu Zheng* \\ Henan Key Lab Spatial Infor. Appl. Eco-environmental Protection, Zhengzhou, China
}

\begin{abstract}
In this work, the temporal-spatial dynamic variation of vegetation coverage from 2010 to 2019 in Urad Grassland has been explored by remote sensing technique. The change of precipitation and temperature has obvious effects on the vegetation, which affects the agriculture and the stability of the ecosystem. Pixel dichotomy approach and correlation analysis are introduced to analyse the temporal and spatial evolution trend of vegetation coverage and its response to climate change in the past 10 years. Specifically, the correlation between vegetation coverage and the critical climate impacting factors such as temperature and precipitation are fully investigated. The results show the vegetation coverage in the study area was influenced by effectors including climate, topography, human activities and government policies. The average annual vegetation coverage showed a downward trend in general from 2010 to 2019. Statistical correlation analysis indicates that the correlation between the vegetation coverage and the precipitation is positive in most parts of the area, while the correlation between the vegetation coverage and the precipitation shows negative with difference with various geographic features. As a result, precipitation was the major natural factor that affected dynamics of vegetation coverage in Urad grassland.
\end{abstract}

\section{Introduction}

Urad Grassland is located in Bayan Nur, Inner Mogoliar, in the extreme arid region where the desert steppe has extremely fragile ecological environment, serious grassland degradation and desertification, and great difficulty in ecological restoration, long restoration term and high cost. At present, over $70 \%$ grassland is seriously degraded, which is the main sand source of sandstorm and poses a threat to ecological security in North China [1]. The study area is the ecological functional area of the northern foot of Yinshan Mountain, which is defined in the National Main Functional Area Plan issued by the State Council in 2011[2]. Therefore, it is not only a typical ecological fragile zone which is very sensitive to global change, but also an important ecological barrier for the mainland.

In nature, vegetation plays a critical role in global change and terrestrial ecosystem response [3]-[5]. In arid areas, vegetation can also be used as a reference index to evaluate the degree of land deg-radation and desertification. Remote sensing techniques are widely used as a useful tool to evaluate and analyze desertification areas for its advantages of

* Corresponding author: Zhangyueying001@126.com 
diverse temporal and spatial data products, low cost and flexibility. Specifically, pixel dichotomy model [6][7] is often adopted for vegetation coverage and desertification monitoring.

As a key aspect of global change, climate change has an important impact on vegetation coverage, and a large number of studies have explored the correlation between vegetation and climate from different time and space scales [5] [7] [8]. Besides climate's respond, other factors such as government policies on protecting grassland and human activities also exert big influences on vegetation change. As an indispensable wind-proof and sand-fixing area in ecological functional area, Urad grassland was suffering serious digression. Accordingly, government has constantly funded billions of dollars in total to encourage in closing grassland, restoring vegetation, returning grazing to grassland and reducing population density since 2003 [1]. However, from the study of vegetation coverage in the grassland ecological function area at the northern foot of Yinshan Mountain from 2000 to 2010 , it indicates the overall fractional vegetation cover showed a fluctuant decrease from 2000 to 2010 [9]. In addition, very few work has carried out with vegetation trend and its response in Urad Grassland that can be referred.

The contributions of this paper can be summarized:

1. Remote sensing dynamic monitoring and detailed analysis of vegetation coverage in the spatial and temporal perspective in Urad grassland for the past ten years are carried out.

2. Correlation between vegetation coverage and climate conditions is further investigated by combining the data of temperature and rainfall, so as to fully understand the vegetation change in this area and reveal its dynamic development trend.

3. Discussions on various inflecting factors are conducted to provide reasonable basis and important data supports for vegetation restoration and ecological environment protection in Urad Grassland.

\section{Study area and approaches}

\subsection{Study area}

Urad Grassland is located in the north of Inner Mongolia, belonging to the city of Bayan Nur with a total area of $6.4 \times 104 \mathrm{~km} 2$ and a population of 1.76 million. Urad Grassland borders Mongolia in the north, Yinshan Mountain in the south, Alxa League in the west and Baotou City in the east. The geographical location of the selected study area is $105^{\circ} 11^{\prime} \mathrm{E} \sim$ $109^{\circ} 52^{\prime} \mathrm{E}, 40^{\circ} 27^{\prime} \mathrm{N} \sim 42^{\circ} 27^{\prime} \mathrm{N}$, covering a total area of $5.48 \times 104 \mathrm{~km} 2$. The average annual air temperature ranges from $7{ }^{\circ} \mathrm{C}$ to $12^{\circ} \mathrm{C}$. Most areas are in arid and semi-arid climate with annual rainfall of 90-300mm and sunshine hours of 3,100-3,300 hours.

Dessert steppe is the dominant land cover in this natural grassland suffering serious degradation [9]. The grassland slopes from northwest to southeast, as the grassland types from east to west are dry grassland, desertification grassland and desert grassland, in which $86.6 \%$ belongs to desert and semi-desert grassland. The study area composes of 3 Banners, namely Urad Front Banner, Urad Middle Banner and Urad Rear Banner, most of which are degraded grassland.

The grassland environment in Urad grassland is fragile, and the vegetation coverage is low with simple ecosystem structure, weak self-regulation and recovery ability. With the fast-economic developments and the variation of habitats, desertification is an increasing threat for utilizing the sustainable grass-land in this region, since the open ecosystem is affected seriously by natural climate change and human activities, especially the phenomena of indiscriminate mining, digging and deforestation, which exert great pressure on the ecological environment leading to grassland degradation. 


\subsection{Remote sensing approaches}

\section{Vegetation coverage via pixel dichotomy}

NDVI is an excellent well-known indicator of plant growth status due to its significant linear correlation with vegetation distribution density and strong ability to detect vegetation with low coverage. However, NDVI has limitation in high vegetation since the indicator saturates at some point and cannot accurately estimate vegetation coverage at this case.

In this study, the widely used pixel dichotomy model [6][7] is applied to indicate vegetation coverage, which extends its feasibility area and improves performance by modifying NDVI. According to the principle of pixel dichotomy model, the NDVI value of a pixel can be expressed as two parts: the information contributed by green vegetation and the information contributed by bare soil. Vegetation coverage can be calculated as:

$$
f_{v c}=\left(N D V I-N D V I_{s o i l}\right) /\left(N D V I_{v e g}-N D V I_{s o i l}\right)
$$

in which, $f_{v c}$ is the vegetation coverage value of the study area; $N D V I_{\text {soil }}$ is NDVI value of bare soil or area without vegetation coverage, which is obtained from NDVI with cumulative percentage of $5 \% . N D V I_{v e g}$ is the NDVI value of pixels completely covered by vegetation, that is, NDVI with cumulative percentage of $95 \%$. The detailed temporal and spatial analysis on vegetation coverage and its trend from 2010 to 2019 can be found in [6].

In this paper, we further this work and the correlation between vegetation coverage and critical climate factors such as precipitation and air temperature are analysed. In order to fully investigate the vegetation growth in Urad grassland, the vegetation coverage statistics were carried out in the growing season with relatively good vegetation quality (July and August), and the annual average vegetation coverage can be obtained in the study area. The response of vegetation cover change to climate factors is analyzed by correlation analysis method [3-5]. The spatial relationship between vegetation cover and precipitation $(\mathrm{mm})$ or air temperature $\left({ }^{\circ} \mathrm{C}\right)$ is analyzed by grid calculator of ArcGIS 10.5, and the significance test is then carried out.

$$
R_{x y}=\sum_{i=1}^{n}\left[\left(x_{i}-\bar{x}\right)\left(y_{i}-\bar{y}\right)\right] / \sqrt{\sum_{i=1}^{n}\left(x_{i}-\bar{x}\right)^{2} \sum_{i=1}^{n}\left(y_{i}-\bar{y}\right)^{2}}
$$

in which, $R_{x y}$ denotes coefficient; $x_{i}$ and is the vegetation cover in the $i$ th year, and $\bar{x}$ is the average of vegetation coverage in 10 years from 2010 to 2019. $y_{i}$ stands for the precipitation $(\mathrm{mm})$ or air temperature $\left({ }^{\circ} \mathrm{C}\right)$ in the $i$ th year; and $\bar{y}$ is the average precipitation or air temperature in 10 years from 2010 to 2019.

\section{Results analysis}

\subsection{Temporal and spatial variation of vegetation coverage}

The spatial distribution of average vegetation in terms of 5 grades from 2010 to 2019 of the study areas can be seen as Fig. 1. It shows that vegetation coverage is generally characterized by high in the east and low in the west and decreasing from east to west. The average vegetation coverage of the study area in 10 years is 0.46 . 


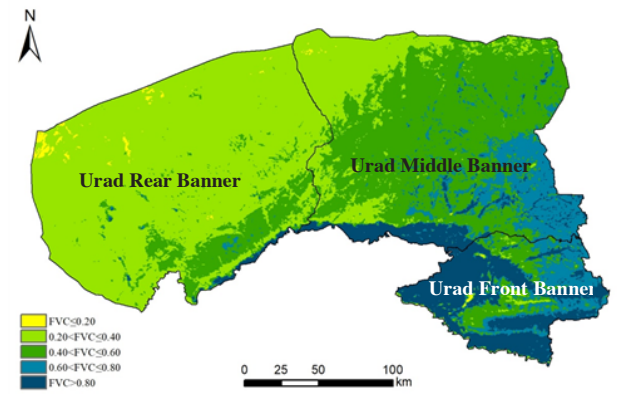

Fig. 1. Spatial distribution of average vegetation coverage from 2010 to 2019.

\subsection{Correlation analysis between vegetation coverage and meteorological factors}

Many factors affecting vegetation coverage including water and heat conditions, soil properties, etc., among which temperature and precipitation plays important roles in the growth of vegetation [4] [5]. Based on the meteorological data of Urad grassland from the last 10 years, the correlation between vegetation coverage and air temperature and precipitation is calculated by ArcGIS 10. 5 grid calculator shown as Fig. 2. To further understand the correlation among different areas, statistics between vegetation coverage and air temperature and precipitation in 3 Banners are depicted as Fig. 3 and Fig. 4.
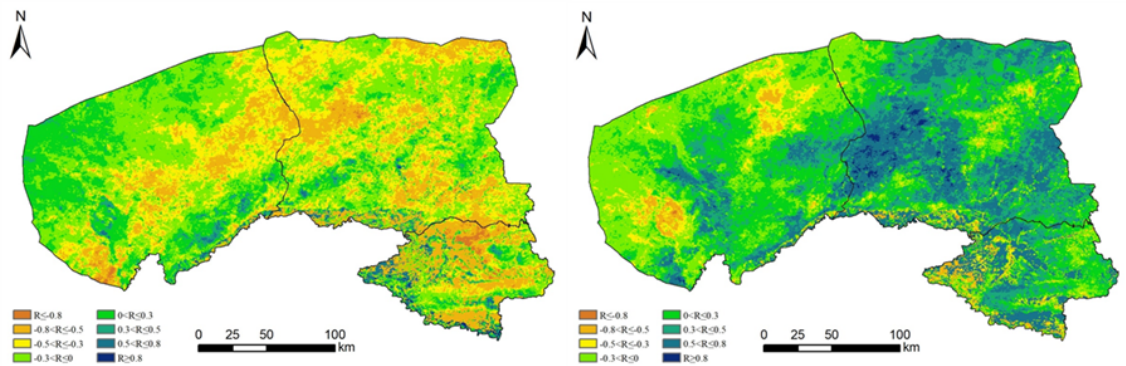

(b) Spatial correlation between vegetation coverage and precipitation

Fig. 2. Spatial correlation between vegetation coverage and temperature/precipitation in Urad grassland over $2010-2019$.

The statistical results show that the average correlation coefficients between vegetation coverage and air temperature and precipitation in the study area are 0.47 and -0.32 , respectively. The areas where vegetation coverage is positively related to air temperature and rainfall are $21.54 \%$ and $85.52 \%$ respectively, indicating that vegetation coverage has a good correlation with precipitation, which is consistent with the research conclusion of Renping Zhang et al. [5] that there is a strong positive correlation between vegetation coverage and rainfall in Inner Mongolia.

From Table 4, area with negative correlation between vegetation coverage and air temperature takes up $78.46 \%$ of the total area, of which $36.01 \%$ and $24.13 \%$ has a low and moderately negative correlation with air temperature and is distributed in the whole study area. $18.32 \%$ of the areas were highly negatively correlated with air temperature mainly distributed in Urad Front Banner. This is because the precipitation is relatively small in dry and semi-arid areas, and the increase of temperature makes the soil water evaporation and plant transpiration increase, while the soil water content decreases, which leads to soil 
drying and hinder the growth of vegetation [9]. 21.54\% of the areas have a positive correlation with air temperature, and most of them have a low positive correlation.

Table 1. Spatial correlation distribution between vegetation coverage and temperature/ precipitation in view of graded correlation coefficient in Urad grassland from 2010 to 2019.

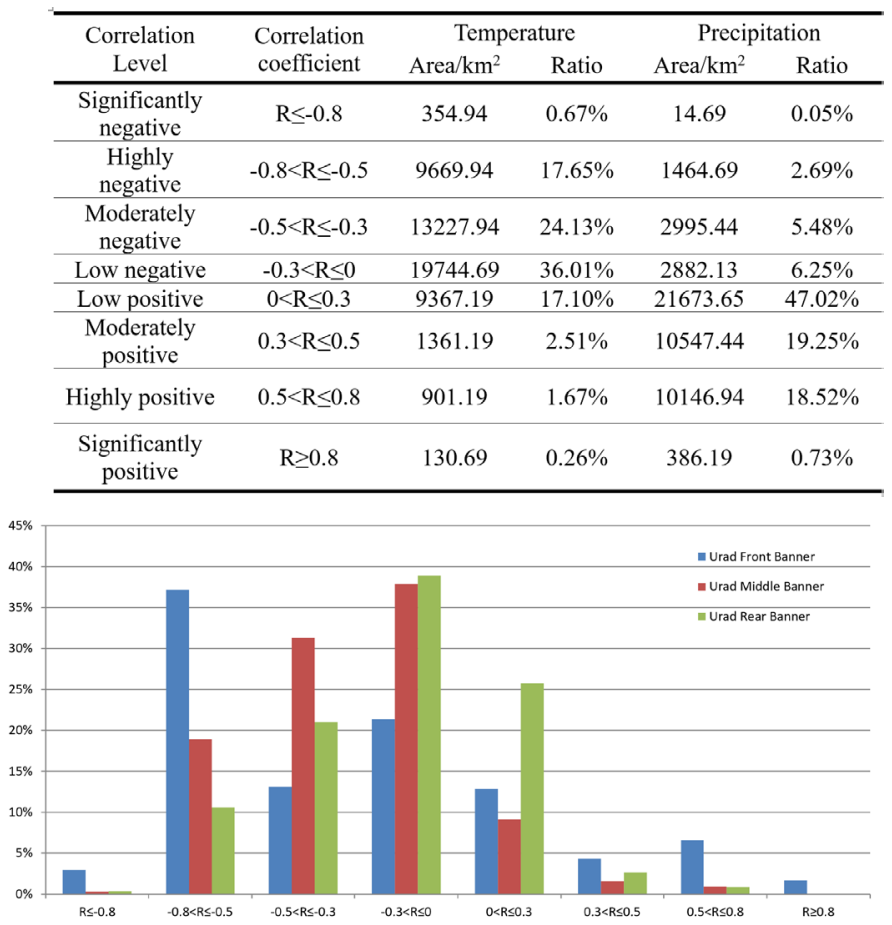

Fig. 3. Ratio comparisons with correlation coefficient between vegetation coverage and temperature in different areas.

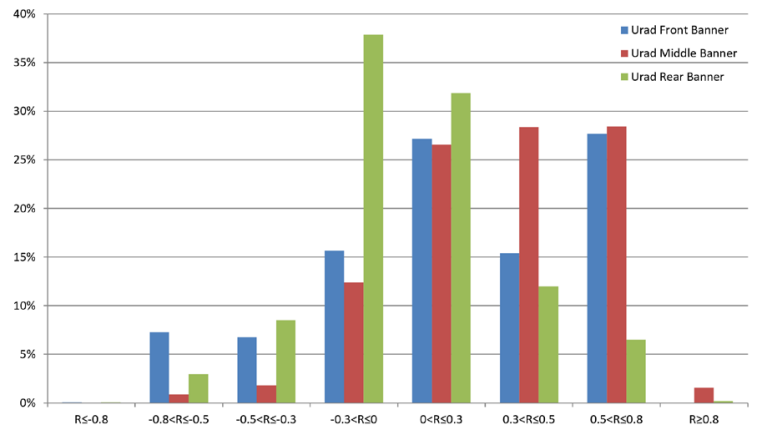

Fig. 4. Ratio comparisons with correlation coefficient between vegetation coverage and precipitation in different areas.

The increase of precipitation is the main natural determinant of vegetation coverage. The area with positive correlation between vegetation coverage and precipitation accounts for $85.52 \%$ of the total Urad grassland, of which $47.02 \%$ has a low positive correlation with precipitation. $19.25 \%$ of the area has a moderate positive correlation with precipitation, as $18.52 \%$ and $0.73 \%$ of the areas have a highly and significantly positive correlation with precipitation. These two areas are mainly distributed in Urad Front Banner and Urad Middle Banner with coefficient of 0.47 and 0.48 , respectively. However, over 
$49 \%$ of Urad Rear Banner have negative correlation with precipitation. The exception may root in the fact that vegetation coverage is affected by human disturbance, topography, soil conditions and many other factors besides climatic conditions.

\section{Conclusions}

In this study, we employ remote sensing techniques to explore the vegetation coverage distribution in view of NDVI over the last ten years. Furthermore, correlation between vegetation coverage and meteorological factors in terms of average temperature and precipitation have been analysed with attempts to seek for helpful suggestions and useful references on ecological reconstruction of Inner Mongolia grassland. From statistical results, we find out:

From 2010 to 2019 , the vegetation coverage in the study area generally shows a slightly downward trend in terms of time change. The obvious degradation areas are mainly distributed in Urad Middle Banner and Urad Rear Banner, which are seriously disturbed by human activities. According to the types and distribution characteristics of degraded grassland in Urad grassland, a serial of implementations supported by national and professional funds have been carried out since 2002 with the aim of promoting the sustainable development of grassland ecology, such as enclosure of degraded grassland, balance control of grass and grazing and supplementary sowing control. The vegetation coverage in Urad Rear Banner remains almost unchanged thanks to the protection encouragement policies from Government. Correlation between vegetation coverage and air temperature was not obvious, showing a negative correlation in most parts, but it also shows difference with geographic locations. The vegetation coverage in the study area is more closely related to precipitation, which further indicates that precipitation is the main natural factor affecting the vegetation coverage change in most of Urad Grassland except Urad Rear Banner. It suggests unlike 10 years before climate factors were the main cause for vegetation change [9], human inventions like indiscriminate mining and overload grazing have become the dominant factors in the change of desertification situation in Urad Rear Banner.

This research is supported by the Sixth Youth Fund for the High-Resolution Project, National Highresolution Project for Provincial Industrialized Application (80-Y40G01-0027-18/20), Zhengzhou Science and Technology Project for Benevolence 2020.

\section{References}

1. Information on http://www.bynrzx.gov.cn/html/106/4437.html

2. Information on http://www.gov.cn/zwgk/2011-06/08/content_1879180.htm

3. L. Su, H. Wang, et al., J Green Sci. Tech. 12, (2020).

4. Z. Wang, X. Deng, et al., Catena, 150, (2017).

5. R. Zhang, Q. Feng, et al., J Desert Research. 35, 5, (2015).

6. Y. Zhang, T. Liu, Y. Wang, et al. CSCNS 2020 (to be published)

7. J. Bai, L. Di, et al., J Sel. Topics Appl. Earth Observ. \& Remote Sens., 7, 11, (2014).

8. J. Bai, et al., In Proceeding of Agro-Geoinformatics, Fairfax, VA, (2013).

9. Y. Zhao, D. Su, et al., Res. Environ. Sci. 30, 2, (2017). 